\title{
On decay properties of solutions for degenerate Kirchhoff equations with strong damping and source terms
}

Shun-Tang $\mathrm{Wu}^{*}$

\section{"Correspondence:} stwu@ntut.edu.tw General Education Center, National Taipei University of Technology,

Taipei, 116, Taiwan

\begin{abstract}
We investigate the degenerate Kirchhoff equations with strong damping and source terms of the form

$$
\varepsilon u_{t t}-\|\nabla u\|_{2}^{2 \gamma} \Delta u-\Delta u_{t}=f(u)
$$

in a bounded domain. We obtain the optimal decay rate for $\left\|\nabla u_{t}\right\|_{2}^{2}$ by deriving its decay estimate from below, provided that either $\varepsilon$ is suitably small or the initial data satisfy the proper smallness assumption. The key ingredient in the proof is based on the work of Ono (J. Math. Anal. Appl. 381(1):229-239, 2011), with necessary modification imposed by our problem.

MSC: 35L70; 35L80
\end{abstract}

Keywords: strong damping; degenerate; Kirchhoff equation; decay estimates

\section{Introduction}

In this paper, we consider the initial boundary value problem for the following degenerate Kirchhoff equations with strong damping and source terms:

$$
\begin{aligned}
& \varepsilon u_{t t}-\|\nabla u\|_{2}^{2 \gamma} \Delta u-\Delta u_{t}=f(u), \quad \text { in } \Omega \times(0, \infty), \\
& u(x, 0)=u_{0}(x), \quad u_{t}(x, 0)=u_{1}(x), \quad x \in \Omega, \\
& u(x, t)=0, \quad x \in \partial \Omega, t \geq 0,
\end{aligned}
$$

where $\Omega$ is a bounded domain in $R^{N}(N \geq 1)$ with a smooth boundary $\partial \Omega$. Here, $\varepsilon>0$ and $\gamma>0$ are positive constants and $f(u)$ is a nonlinear term like $|u|^{p-1} u, p>1$.

In the case $N=1$, the nonlinear vibrations of the elastic string are written in the form:

$$
\rho h \frac{\partial^{2} u}{\partial t^{2}}=\left\{p_{0}+\frac{E h}{2 L} \int_{0}^{L}\left(\frac{\partial u}{\partial x}\right)^{2} d x\right\} \frac{\partial^{2} u}{\partial x^{2}}+f
$$

for $0<x<L, t \geq 0$; where $u$ is the lateral deflection, $x$ the space coordinate, $t$ the time, $E$ the Young modulus, $\rho$ the mass density, $h$ the cross section area, $L$ the length, $p_{0}$ the initial axial tension and $f$ the external force. The equation was first introduced by Kirchhoff [1] in the study of stretched strings and plates, and is called the wave equation of Kirchhoff type

\section{Springer}

(c) $2012 \mathrm{Wu}$; licensee Springer. This is an Open Access article distributed under the terms of the Creative Commons Attribution License (http://creativecommons.org/licenses/by/2.0), which permits unrestricted use, distribution, and reproduction in any medium, provided the original work is properly cited. 
after his name. Moreover, it is said that (1.4) is degenerate if $p_{0}=0$ and nondegenerate if $p_{0}>0$.

A number of results on the solutions to problem (1.1)-(1.3) have been established by many authors. For example, Hosoya and Yamada [2] studied the following equation:

$$
u_{t t}-M\left(\|\nabla u\|_{2}^{2}\right) \Delta u+\delta u_{t}=0
$$

with $M(r) \geq M_{0}>0$ (the nondegenerate case). They proved the global existence of a unique solution under the small data condition in $H^{2}(\Omega) \cap H_{0}^{1}(\Omega) \times H_{0}^{1}(\Omega)$. Similarly, in the degenerate case $\left(M(r)=r^{\gamma}\right)$, Nishihara and Yamada [3] obtained the global existence of solutions for small initial data in $H^{2}(\Omega) \cap H_{0}^{1}(\Omega) \times H_{0}^{1}(\Omega)$.

Concerning decay properties of solutions, Nakao [4] has derived decay estimates from above of solutions when $f(u)=0$ in (1.1). Later, Nishihara [5] established a decay estimate from below of the potential of solutions to problem (1.1) without imposing $f(u)$. Nishihara and Ono [6-8] studied detailed cases of nondegenerate type and degenerate type to problem (1.1)-(1.3). They proved the existence and uniqueness of a global solution for initial data $\left(u_{0}, u_{1}\right) \in H^{2}(\Omega) \cap H_{0}^{1}(\Omega) \times L^{2}(\Omega)$ and the polynomial decay of the solution. Recently, in the absence of $f(u)$ in (1.1), Ono [9] proved the optimal decay estimate for $\|\nabla u\|_{2}^{2}$. And the decay property of the norm $\|\Delta u\|_{2}^{2}$ for $t \geq 0$ was also given in that paper.

Motivated by these works, in this paper, we intend to give the optimal decay estimate for $\left\|\nabla u_{t}\right\|_{2}$ to problem (1.1)-(1.3). In this way, we can extend the result in [8] where the author considered (1.1) with $\gamma \geq 1$; and we also improve the result of [9] in the presence of a nonlinear source term. We followed the technique skill introduced in [9] with the concept of a stable set in $H^{2}(\Omega) \cap H_{0}^{1}(\Omega)$ to derive the optimal decay rate of $\left\|\nabla u_{t}\right\|_{2}$. The content of this paper is organized as follows. In Section 2, we give some lemmas which will be used later. In Section 3, we derive the global solution and its decay properties with $\gamma>0$.

\section{Preliminary results}

In this section, we shall give some lemmas which will be used throughout this work. We denote by $\|\cdot\|_{p}$ the $L^{p}$ norm over $\Omega$.

Lemma 2.1 (The Sobolev-Poincaré inequality) If $2 \leq p \leq \frac{2 N}{N-2}(2 \leq p<\infty$ if $N=1,2)$, then

$$
\|u\|_{p} \leq B_{1}\|\nabla u\|_{2}, \quad \text { for } u \in H_{0}^{1}(\Omega)
$$

holds with some positive constant $B_{1}$.

Lemma 2.2 $([4,9])$ Let $\phi(t)$ be a nonincreasing and nonnegative function on $[0, \infty)$ such that

$$
\phi(t)^{1+r} \leq \omega_{0}(\phi(t)-\phi(t+1))
$$

with certain constants $\omega_{0} \geq 0$ and $r>0$. Then, the function $\phi(t)$ satisfies

$$
\phi(t) \leq\left(\phi(0)^{-r}+\omega_{0}^{-1} r[t-1]^{+}\right)^{-\frac{1}{r}}
$$

for $t \geq 0$, where we put $[a]^{+}=\max \{0, a\}$ and $\frac{1}{[a]^{+}}=\infty$ if $[a]^{+}=0$. 
Now, we state the local existence for problem (1.1)-(1.3) which can be established by the arguments of [8].

Theorem 2.3 Suppose that $\left(u_{0}, u_{1}\right) \in H^{2}(\Omega) \cap H_{0}^{1}(\Omega) \times L^{2}(\Omega)$, then there exists a unique solution $u$ of problem (1.1)-(1.3) satisfying

$$
\begin{aligned}
& u(t) \in C\left([0, T) ; H^{2}(\Omega) \cap H_{0}^{1}(\Omega)\right), \\
& u_{t}(t) \in C\left([0, T) ; L^{2}(\Omega)\right) \cap L^{2}\left(0, T ; H_{0}^{1}(\Omega)\right) .
\end{aligned}
$$

Moreover, at least one of the following statements holds true:

(i) $T=\infty$,

(ii) $\left\|u_{t}\right\|_{2}^{2}+\|\Delta u\|_{2}^{2} \rightarrow \infty$, as $t \rightarrow T^{-}$.

\section{Decay properties}

In this section, we shall show the decay properties of solutions $u$ to problem (1.1)-(1.3). For this purpose, we define

$$
\begin{aligned}
& I(t) \equiv I(u(t))=\|\nabla u(t)\|_{2}^{2(\gamma+1)}-\|u(t)\|_{p+1}^{p+1}, \\
& J(t) \equiv J(u(t))=\frac{1}{2(\gamma+1)}\|\nabla u(t)\|_{2}^{2(\gamma+1)}-\frac{1}{p+1}\|u(t)\|_{p+1}^{p+1},
\end{aligned}
$$

and the energy function

$$
E(t)=\frac{\varepsilon}{2}\left\|u_{t}\right\|_{2}^{2}+J(t)
$$

for $u(t) \in H_{0}^{1}(\Omega), t \geq 0$. Then, multiplying (1.1) by $u_{t}$ and integrating it over $\Omega$, we see that

$$
E^{\prime}(t)=-\left\|\nabla u_{t}\right\|_{2}^{2}
$$

Lemma 3.1 Let $\left(u_{0}, u_{1}\right) \in H^{2}(\Omega) \cap H_{0}^{1}(\Omega) \times L^{2}(\Omega)$. Suppose that $p$ and $\gamma$ satisfy

$$
p>2 \gamma+1 \quad \text { and } \quad 1<p \leq \frac{N+2}{N-2} \quad(p<\infty, \text { if } N=1,2) .
$$

If $I(0)>0$ and

$$
l=B_{1}^{p+1}\left(\frac{2(p+1)(\gamma+1)}{p-2 \gamma-1} E(0)\right)^{\frac{p-2 \gamma-1}{2(\gamma+1)}}<1,
$$

then $I(t)>0$, for $t \in[0, T]$.

Proof Since $I(0)>0$, then there exists $t_{\max }<T$ such that

$$
I(t) \geq 0, \quad t \in\left[0, t_{\max }\right],
$$

which gives

$$
J(t)=\frac{p-2 \gamma-1}{2(p+1)(\gamma+1)}\|\nabla u(t)\|_{2}^{2(\gamma+1)}+\frac{1}{p+1} I(t) \geq \frac{p-2 \gamma-1}{2(p+1)(\gamma+1)}\|\nabla u(t)\|_{2}^{2(\gamma+1)} .
$$


Thus, from (3.3) and $E(t)$ being nonincreasing by (3.4), we have that

$$
\begin{aligned}
\|\nabla u(t)\|_{2}^{2(\gamma+1)} & \leq \frac{2(p+1)(\gamma+1)}{p-2 \gamma-1} J(t) \leq \frac{2(p+1)(\gamma+1)}{p-2 \gamma-1} E(t) \\
& \leq \frac{2(p+1)(\gamma+1)}{p-2 \gamma-1} E(0) \text { on }\left[0, t_{\max }\right] .
\end{aligned}
$$

And so, exploiting Lemma 2.1, (3.8) and (3.6), we obtain

$$
\begin{aligned}
\|u\|_{p+1}^{p+1} & \leq B_{1}^{p+1}\|\nabla u\|_{2}^{p+1} \leq B_{1}^{p+1}\left(\frac{2(p+1)(\gamma+1)}{p-2 \gamma-1} E(0)\right)^{\frac{p-2 \gamma-1}{2(\gamma+1)}}\|\nabla u(t)\|_{2}^{2(\gamma+1)} \\
& =l\|\nabla u(t)\|_{2}^{2(\gamma+1)}<\|\nabla u(t)\|_{2}^{2(\gamma+1)} \text { on }\left[0, t_{\max }\right] .
\end{aligned}
$$

Therefore,

$$
I(t)=\|\nabla u(t)\|_{2}^{2(\gamma+1)}-\|u(t)\|_{p+1}^{p+1}>0 \quad \text { on }\left[0, t_{\max }\right]
$$

By repeating this procedure and using the fact that

$$
\lim _{t \rightarrow t_{\max }} B_{1}^{p+1}\left(\frac{2(p+1)(\gamma+1)}{p-2 \gamma-1} E(t)\right)^{\frac{p-2 \gamma-1}{2(\gamma+1)}} \leq l<1
$$

we can take $t_{\max }=T$.

Lemma 3.2 Let $u$ satisfy the assumptions of Lemma 3.1. Then there exists $0<\eta<1$ such that

$$
\|u(t)\|_{p+1}^{p+1} \leq(1-\eta)\|\nabla u(t)\|_{2}^{2(\gamma+1)} \text { for } t \in[0, T]
$$

with $\eta=1-l$.

Proof From (3.9), we get

$$
\|u(t)\|_{p+1}^{p+1} \leq l\|\nabla u(t)\|_{2}^{2(\gamma+1)}, \quad \text { for } t \in[0, T]
$$

Let $\eta=1-l$, then we have the inequality (3.10).

Theorem 3.3 Suppose that $\left(u_{0}, u_{1}\right) \in H^{2}(\Omega) \cap H_{0}^{1}(\Omega) \times L^{2}(\Omega), I(0)>0$, (3.5) and (3.6) hold. Then there exists a unique global solution $u$ of problem (1.1)-(1.3) satisfying

$$
\begin{aligned}
& u(t) \in C\left([0, \infty) ; H^{2}(\Omega) \cap H_{0}^{1}(\Omega)\right) \cap L^{\infty}\left(0, \infty ; H^{2}(\Omega) \cap H_{0}^{1}(\Omega)\right), \\
& u_{t}(t) \in C\left([0, \infty) ; L^{2}(\Omega)\right) \cap L^{2}\left(0, \infty ; H_{0}^{1}(\Omega)\right) \cap L^{\infty}\left(0, \infty ; L^{2}(\Omega)\right) .
\end{aligned}
$$

Furthermore, we have the following decay estimates:

$$
E(t) \leq\left(E(0)^{-\frac{\gamma}{\gamma+1}}+\frac{\gamma}{\gamma+1}\left(\alpha E(0)^{\frac{\gamma}{2(\gamma+1)}}+\beta\right)^{-2}[t-1]^{+}\right)^{-\frac{\gamma+1}{\gamma}} \quad \text { for } t \geq 0,
$$

where $\alpha$ and $\beta$ are some positive constants given by (3.15). 
Proof First, for $T=\infty$, we can obtain the result by following the arguments as in [8], so we omit the proof. Next, we will show the decay estimate. For $t \geq 0$, integrating (3.4) over $[t, t+1]$, we have

$$
\int_{t}^{t+1}\left\|\nabla u_{t}(s)\right\|_{2}^{2} d s=E(t)-E(t+1) \equiv D(t)^{2} .
$$

Then there exist $t_{1} \in\left[t, t+\frac{1}{4}\right]$ and $t_{2} \in\left[t+\frac{3}{4}, t+1\right]$ such that

$$
\left\|\nabla u_{t}\left(t_{i}\right)\right\|_{2}^{2} \leq 4 D(t)^{2}, \quad i=1,2 .
$$

Multiplying (1.1) by $u$, integrating it over $\Omega \times\left[t_{1}, t_{2}\right]$, using integration by parts and Lemma 2.1, we get

$$
\begin{aligned}
\int_{t_{1}}^{t_{2}}\left(\|\nabla u(s)\|_{2}^{2(\gamma+1)}-\|u(s)\|_{p+1}^{p+1}\right) d s \\
\leq \varepsilon B_{1}^{2} \int_{t}^{t+1}\left\|\nabla u_{t}\right\|_{2}^{2} d s+\varepsilon B_{1}^{2} \sum_{i=1}^{2}\left\|\nabla u_{t}\left(t_{i}\right)\right\|_{2}\left\|\nabla u\left(t_{i}\right)\right\|_{2} \\
\quad+\int_{t}^{t+1}\left\|\nabla u_{t}\right\|_{2}\|\nabla u\|_{2} d s .
\end{aligned}
$$

Hence, from the definition of $I(t)$ by (3.1), using (3.12), (3.13) and (3.8), we obtain

$$
\int_{t_{1}}^{t_{2}} I(s) d s \leq \varepsilon B_{1}^{2} D(t)^{2}+\left(4 \varepsilon B_{1}^{2}+1\right) D(t)\left(\frac{2(p+1)(\gamma+1)}{p-2 \gamma-1} E(t)\right)^{\frac{1}{2(\gamma+1)}} .
$$

On the other hand, integrating (3.4) over $\left[t, t_{2}\right]$, noting that $E\left(t_{2}\right) \leq 2 \int_{t_{1}}^{t_{2}} E(t) d t$ due to $t_{2}-t_{1} \geq \frac{1}{2}$, and using (3.3) and (3.7), we have that

$$
\begin{aligned}
E(t)= & E\left(t_{2}\right)+\int_{t}^{t_{2}}\left\|\nabla u_{t}(s)\right\|_{2}^{2} d s \\
\leq & 2 \int_{t_{1}}^{t_{2}} E(s) d s+\int_{t}^{t_{2}}\left\|\nabla u_{t}(s)\right\|_{2}^{2} d s \\
\leq & \left(1+\varepsilon B_{1}^{2}\right) \int_{t}^{t_{2}}\left\|\nabla u_{t}(s)\right\|_{2}^{2} d s+\frac{p-2 \gamma-1}{(p+1)(\gamma+1)} \int_{t}^{t_{2}}\|\nabla u(s)\|_{2}^{2(\gamma+1)} d s \\
& +\frac{2}{(p+1)} \int_{t}^{t_{2}} I(s) d s .
\end{aligned}
$$

We then use the fact that $\|\nabla u(t)\|_{2}^{2(\gamma+1)} \leq \frac{1}{1-l} I(t)$ by (3.10), (3.12) and (3.14) to obtain

$$
\begin{aligned}
E(t) & \leq\left(1+\varepsilon B_{1}^{2}\right) D(t)^{2}+c_{1} \int_{t}^{t_{2}} I(t) d t \\
& \leq \alpha D(t)^{2}+\beta D(t) E(t)^{\frac{1}{2(\gamma+1)}},
\end{aligned}
$$

where

$$
\alpha=\left(1+\varepsilon B_{1}^{2}+c_{1} \varepsilon B_{1}^{2}\right) \quad \text { and } \quad \beta=c_{1}\left(4 \varepsilon B_{1}^{2}+1\right)\left(\frac{2(p+1)(\gamma+1)}{p-2 \gamma-1}\right)^{\frac{1}{2(\gamma+1)}}
$$


with

$$
c_{1}=\frac{p-2 \gamma-1}{(p+1)(\gamma+1)(1-l)}+\frac{2}{(p+1)} .
$$

Moreover, from (3.12) and $E(t)$ being nonincreasing by (3.4), we note that

$$
D(t) \leq E^{\frac{1}{2}}(t) \leq E(0)^{\frac{\gamma}{2(\gamma+1)}} E(t)^{\frac{1}{2(\gamma+1)}} .
$$

Thus, by Young's inequality, (3.15) becomes

$$
\begin{aligned}
E(t) & \leq\left(\alpha E(0)^{\frac{\gamma}{2(\gamma+1)}}+\beta\right) D(t) E(t)^{\frac{1}{2(\gamma+1)}} \\
& \leq \frac{2 \gamma+1}{2(\gamma+1)}\left[\left(\alpha E(0)^{\frac{\gamma}{2(\gamma+1)}}+\beta\right) D(t)\right]^{\frac{2(\gamma+1)}{2 \gamma+1}}+\frac{1}{2(\gamma+1)} E(t) .
\end{aligned}
$$

This implies that

$$
\begin{aligned}
E(t)^{1+\frac{\gamma}{\gamma+1}} & \leq\left(\alpha E(0)^{\frac{\gamma}{2(\gamma+1)}}+\beta\right)^{2} D^{2}(t) \\
& =\left(\alpha E(0)^{\frac{\gamma}{2(\gamma+1)}}+\beta\right)^{2}(E(t)-E(t+1)) .
\end{aligned}
$$

Therefore, applying Lemma 2.2, we conclude that

$$
E(t) \leq\left(E(0)^{-\frac{\gamma}{\gamma+1}}+\frac{\gamma+1}{\gamma}\left(\alpha E(0)^{\frac{\gamma}{2(\gamma+1)}}+\beta\right)^{-2}[t-1]^{+}\right)^{-\frac{\gamma+1}{\gamma}}, \quad \text { for } t \geq 0 .
$$

Remark (i) Based on the estimate (3.11), we have further the following estimates:

$$
\|\nabla u\|_{2}^{2} \leq c_{2}(1+t)^{-\frac{1}{\gamma}}, \quad\left\|u_{t}\right\|_{2}^{2} \leq c_{3}(1+t)^{-1-\frac{1}{\gamma}}, \quad \text { for } t \geq 0
$$

where $c_{2}$ and $c_{3}$ are some positive constants.

(ii) If $\omega>\frac{\gamma}{\gamma+1}$, then

$$
\int_{0}^{t} E(s)^{\omega} d s \leq k_{1} E(0)^{\omega-\frac{\gamma}{\gamma+1}}
$$

with $k_{1}=\frac{(\gamma+1)^{2} \omega-\gamma}{((\gamma+1) \omega-\gamma)(\gamma+1)}\left(\alpha E(0)^{\frac{\gamma}{2(\gamma+1)}}+\beta\right)^{2}$. Indeed, by (3.11), we see that

$$
\begin{aligned}
& \int_{0}^{t} E(s)^{\omega} d s \leq \int_{0}^{t}\left(E(0)^{-\frac{\gamma}{\gamma+1}}+\frac{\gamma+1}{\gamma}\left(\alpha E(0)^{\frac{\gamma}{2(\gamma+1)}}+\beta\right)^{-2}[s-1]^{+}\right)^{-\frac{(\gamma+1) \omega}{\gamma}} d s \\
& =E(0)^{\omega}+\int_{1}^{t}\left(E(0)^{-\frac{\gamma}{\gamma+1}}+\frac{\gamma+1}{\gamma}\left(\alpha E(0)^{\frac{\gamma}{2(\gamma+1)}}+\beta\right)^{-2}(s-1)\right)^{-\frac{(\gamma+1) \omega}{\gamma}} d s .
\end{aligned}
$$

A direct computation yields the result.

Next, we will improve the decay rate for $\left\|u_{t}\right\|_{2}^{2}$ given by (3.17) 
Proposition 3.4 If $\left(u_{0}, u_{1}\right) \in H^{2}(\Omega) \cap H_{0}^{1}(\Omega) \times L^{2}(\Omega)$, then the solution $u$ of problem (1.1)(1.3) satisfies

$$
\left\|u_{t}\right\|_{2}^{2} \leq c_{4}(1+t)^{-2-\frac{1}{\gamma}} \quad \text { and } \quad \int_{0}^{t}\left\|\nabla u_{t}\right\|_{2}^{2} d s \leq c_{5}, \quad \text { for } t \geq 0
$$

where $c_{4}$ and $c_{5}$ are some positive constants.

Proof Multiplying (1.1) by $u_{t}$ and integrating it over $\Omega$, we have

$$
\frac{\varepsilon}{2} \frac{d}{d t}\left\|u_{t}\right\|_{2}^{2}+\left\|\nabla u_{t}\right\|_{2}^{2}=-\|\nabla u\|_{2}^{2 \gamma} \int_{\Omega} \nabla u \nabla u_{t} d x+\int_{\Omega}|u|^{p-1} u u_{t} d x .
$$

We now estimate the right-hand side of (3.20). Employing Hölder's inequality and Young's inequality, the first term gives

$$
\begin{aligned}
\|\nabla u\|_{2}^{2 \gamma} \int_{\Omega} \nabla u \nabla u_{t} d x & \leq\|\nabla u\|_{2}^{2 \gamma+1}\left\|\nabla u_{t}\right\|_{2} \\
& \leq\|\nabla u\|_{2}^{2(2 \gamma+1)}+\frac{1}{4}\left\|\nabla u_{t}\right\|_{2}^{2} .
\end{aligned}
$$

As for the second term, using Hölder's inequality, Lemma 2.1, Young's inequality and (3.8) yields

$$
\begin{aligned}
\left.\left|\int_{\Omega}\right| u\right|^{p-1} u u_{t} d x \mid & \leq\|u\|_{p+1}^{p}\left\|u_{t}\right\|_{p+1} \leq B_{1}^{p+1}\|\nabla u\|_{2}^{p}\left\|\nabla u_{t}\right\|_{2} \\
& \leq B_{1}^{2(p+1)}\|\nabla u\|_{2}^{2 p}+\frac{1}{4}\left\|\nabla u_{t}\right\|_{2}^{2} \\
& \leq B_{1}^{2(p+1)}\left(\frac{2(p+1)(\gamma+1)}{p-2 \gamma-1} E(0)\right)^{\frac{p-2 \gamma-1}{\gamma+1}}\|\nabla u\|_{2}^{2(2 \gamma+1)}+\frac{1}{4}\left\|\nabla u_{t}\right\|_{2}^{2} .
\end{aligned}
$$

Combining these estimates and using (3.17), we arrive at

$$
\varepsilon \frac{d}{d t}\left\|u_{t}\right\|_{2}^{2}+\left\|\nabla u_{t}\right\|_{2}^{2} \leq c_{6}\|\nabla u\|_{2}^{2(2 \gamma+1)} \leq c_{7}(1+t)^{-2-\frac{1}{\gamma}}
$$

where $c_{6}=2\left(1+B_{1}^{2(p+1)}\left(\frac{2(p+1)(\gamma+1)}{p-2 \gamma-1} E(0)\right)^{\frac{p-2 \gamma-1}{\gamma+1}}\right)$ and $c_{7}=c_{6} c_{2}^{2 \gamma+1}$. Then, from Lemma 2.1, we have

$$
\varepsilon \frac{d}{d t}\left\|u_{t}\right\|_{2}^{2}+B_{1}^{-1}\left\|u_{t}\right\|_{2}^{2} \leq c_{7}(1+t)^{-2-\frac{1}{\gamma}} .
$$

Therefore, the desired estimate (3.19) is obtained.

In order to obtain the decay estimates for problem (1.1)-(1.3), we need the function $H(t)$ and equality (3.22) as in [9]. Define

$$
H(t)= \begin{cases}\varepsilon \frac{\left\|u_{t}\right\|_{2}^{2}}{\|\nabla u\|_{2}^{2 \gamma}}+\|\nabla u\|_{2}^{2}, & \text { if } \gamma \geq 1 ; \\ \varepsilon \frac{\left\|u_{t}\right\|_{2}^{2}}{\|\nabla u\|_{2}^{2}}+\|\nabla u\|_{2}^{2 \gamma}, & \text { if } 0<\gamma<1 .\end{cases}
$$


Multiplying (1.1) by $\frac{2 u_{t}}{\|\nabla u\|_{2}^{2(\gamma+k)}}$ with $k \geq 0$ and integrating it over $\Omega$, we have the following equality:

$$
\begin{aligned}
& \frac{d}{d t}\left[\varepsilon \frac{\left\|u_{t}\right\|_{2}^{2}}{\|\nabla u\|_{2}^{2(\gamma+k)}}+\frac{1}{\|\nabla u\|_{2}^{2(k-1)}}\right]+2 \frac{\left\|\nabla u_{t}\right\|_{2}^{2}}{\|\nabla u\|_{2}^{2(\gamma+k)}} \\
& \quad=-2 \varepsilon(\gamma+k) \frac{\int_{\Omega} \nabla u \nabla u_{t} d x}{\|\nabla u\|_{2}^{2(\gamma+k+1)}}\left\|u_{t}\right\|_{2}^{2}-2 k \frac{\int_{\Omega} \nabla u \nabla u_{t} d x}{\|\nabla u\|_{2}^{2 k}}+2 \frac{\int_{\Omega}|u|^{p-1} u u_{t} d x}{\|\nabla u\|_{2}^{2(\gamma+k)}} .
\end{aligned}
$$

Proposition 3.5 Let $\left(u_{0}, u_{1}\right) \in H^{2}(\Omega) \cap H_{0}^{1}(\Omega) \times L^{2}(\Omega), I(0)>0$, (3.5)-(3.6) hold and $\left\|\nabla u_{0}\right\|_{2}>0$. Suppose that $\|\nabla u(t)\|_{2}>0$ for $0 \leq t<T_{1}$ and

$$
\begin{aligned}
& 1-2 \gamma B_{1}\left(\varepsilon H^{\gamma}(0)\right)^{\frac{1}{2}}>0, \quad \text { if } \gamma \geq 1 ; \\
& 1-2 B_{1}(\varepsilon H(0))^{\frac{1}{2}}>0, \quad \text { if } 0<\gamma<1 .
\end{aligned}
$$

Then it holds that, for $0 \leq t<T_{1}$,

$$
\begin{aligned}
& H(t) \leq H(0)+\alpha_{1} E(0)^{\frac{p-2 \gamma}{\gamma+1}}, \quad \text { if } \gamma \geq 1 ; \\
& H(t) \leq H(0)+\alpha_{2} E(0)^{\frac{\gamma}{\gamma+1}}, \quad \text { if } 0<\gamma<1,
\end{aligned}
$$

where constants $\alpha_{1}$ and $\alpha_{2}$ are given by (3.31) and (3.36).

Proof (i) In the case $\gamma \geq 1$, we observe from (3.21) that

$$
\varepsilon \frac{\left\|u_{t}\right\|_{2}}{\|\nabla u\|_{2}}=\varepsilon^{\frac{1}{2}}\left(\varepsilon \frac{\left\|u_{t}\right\|_{2}^{2}}{\|\nabla u\|_{2}^{2 \gamma}}\|\nabla u\|_{2}^{2 \gamma-2}\right)^{\frac{1}{2}} \leq\left(\varepsilon H^{\gamma}(t)\right)^{\frac{1}{2}}
$$

Using (3.22) with $k=0$ yields

$$
\frac{d}{d t} H(t)+2 \frac{\left\|\nabla u_{t}\right\|_{2}^{2}}{\|\nabla u\|_{2}^{2 \gamma}}=-2 \gamma \varepsilon \frac{\int_{\Omega} \nabla u \nabla u_{t} d x}{\|\nabla u\|_{2}^{2(\gamma+1)}}\left\|u_{t}\right\|_{2}^{2}+2 \frac{\int_{\Omega}|u|^{p-1} u u_{t} d x}{\|\nabla u\|_{2}^{2 \gamma}}
$$

Now, we estimate the right-hand side of (3.28). So, thanks to Hölder's inequality, Lemma 2.1, (3.8) and Young's inequality, we see that

$$
2 \gamma \varepsilon \frac{\int_{\Omega} \nabla u \nabla u_{t} d x}{\|\nabla u\|_{2}^{2(\gamma+1)}}\left\|u_{t}\right\|_{2}^{2} \leq 2 \gamma B_{1} \varepsilon \frac{\left\|u_{t}\right\|_{2}}{\|\nabla u\|_{2}} \frac{\left\|\nabla u_{t}\right\|_{2}^{2}}{\|\nabla u\|_{2}^{2 \gamma}}
$$

and

$$
\begin{aligned}
2 \frac{\int_{\Omega}|u|^{p-1} u u_{t} d x}{\|\nabla u\|_{2}^{2 \gamma}} & \leq 2 B_{1}^{p+1}\|\nabla u\|_{2}^{p-\gamma} \frac{\left\|\nabla u_{t}\right\|_{2}}{\|\nabla u\|_{2}^{\gamma}} \\
& \leq 2 B_{1}^{p+1}\left(\frac{2(p+1)(\gamma+1)}{p-2 \gamma-1} E(t)\right)^{\frac{p-\gamma}{2(\gamma+1)}} \frac{\left\|\nabla u_{t}\right\|_{2}}{\|\nabla u\|_{2}^{\gamma}} \\
& \leq B_{1}^{2(p+1)}\left(\frac{2(p+1)(\gamma+1)}{p-2 \gamma-1} E(t)\right)^{\frac{p-\gamma}{\gamma+1}}+\frac{\left\|\nabla u_{t}\right\|_{2}^{2}}{\|\nabla u\|_{2}^{2 \gamma}} .
\end{aligned}
$$


Taking into account these two estimates in (3.28) and using $\varepsilon \frac{\left\|u_{t}\right\|_{2}}{\|\nabla u\|_{2}} \leq\left(\varepsilon H^{\gamma}(t)\right)^{\frac{1}{2}}$ by (3.27), we obtain

$$
\frac{d}{d t} H(t)+\left(1-2 \gamma B_{1}\left(\varepsilon H^{\gamma}(t)\right)^{\frac{1}{2}}\right) \frac{\left\|\nabla u_{t}\right\|_{2}^{2}}{\|\nabla u\|_{2}^{2 \gamma}} \leq B_{1}^{2(p+1)}\left(\frac{2(p+1)(\gamma+1)}{p-2 \gamma-1} E(t)\right)^{\frac{p-\gamma}{\gamma+1}} .
$$

If $1-2 \gamma B_{1}\left(\varepsilon H^{\gamma}(0)\right)^{\frac{1}{2}}>0$, then there exists $T_{2}$ such that $0<T_{2} \leq T_{1}$ and

$$
1-2 \gamma B_{1}\left(\varepsilon H^{\gamma}(t)\right)^{\frac{1}{2}} \geq 0, \quad \text { for } 0 \leq t \leq T_{2} .
$$

Hence

$$
\frac{d}{d t} H(t) \leq B_{1}^{2(p+1)}\left(\frac{2(p+1)(\gamma+1)}{p-2 \gamma-1} E(t)\right)^{\frac{p-\gamma}{\gamma+1}}
$$

for $0 \leq t \leq T_{2}$. Applying (3.18) with $\omega=\frac{p-\gamma}{\gamma+1}$, we derive that

$$
H(t) \leq H(0)+\alpha_{1} E(0)^{\frac{p-2 \gamma}{\gamma+1}}
$$

with

$$
\alpha_{1}=B_{1}^{2(p+1)}\left(\frac{2(p+1)(\gamma+1)}{p-2 \gamma-1}\right)^{\frac{p-\gamma}{\gamma+1}} \frac{(\gamma+1)(p-\gamma)-\gamma}{(p-2 \gamma)(\gamma+1)}\left(\alpha E(0)^{\frac{\gamma}{2(\gamma+1)}}+\beta\right)^{2} .
$$

Therefore, we see that (3.29)-(3.31) hold true for $0 \leq t<T_{1}$, which gives the estimate (3.25) for $\gamma \geq 1$.

(ii) In the case $0<\gamma<1$, we note from (3.21) that

$$
\varepsilon \frac{\left\|u_{t}\right\|_{2}}{\|\nabla u\|_{2}} \leq(\varepsilon H(t))^{\frac{1}{2}}
$$

From (3.22) with $k=1-\gamma$, we have

$$
\begin{aligned}
\frac{d}{d t} H(t)+2 \frac{\left\|\nabla u_{t}\right\|_{2}^{2}}{\|\nabla u\|_{2}^{2}}= & -2 \varepsilon \frac{\int_{\Omega} \nabla u \nabla u_{t} d x}{\|\nabla u\|_{2}^{4}}\left\|u_{t}\right\|_{2}^{2}-2(1-\gamma) \frac{\int_{\Omega} \nabla u \nabla u_{t} d x}{\|\nabla u\|_{2}^{2(1-\gamma)}} \\
& +2 \frac{\int_{\Omega}|u|^{p-1} u u_{t} d x}{\|\nabla u\|_{2}^{2}}
\end{aligned}
$$

Similar to those estimates, as in the case for $\gamma \geq 1$, the right-hand side of (3.33) can be estimated as follows:

$$
\begin{aligned}
2 \varepsilon \frac{\int_{\Omega} \nabla u \nabla u_{t} d x}{\|\nabla u\|_{2}^{4}}\left\|u_{t}\right\|_{2}^{2} & \leq 2 B_{1} \varepsilon \frac{\left\|u_{t}\right\|_{2}}{\|\nabla u\|_{2}} \frac{\left\|\nabla u_{t}\right\|_{2}^{2}}{\|\nabla u\|_{2}^{2}} \leq 2 B_{1}(\varepsilon H(t))^{\frac{1}{2}} \frac{\left\|\nabla u_{t}\right\|_{2}^{2}}{\|\nabla u\|_{2}^{2}} \\
2(1-\gamma) \frac{\int_{\Omega} \nabla u \nabla u_{t} d x}{\|\nabla u\|_{2}^{2(1-\gamma)}} & \leq 2(1-\gamma)\|\nabla u\|_{2}^{2 \gamma} \frac{\left\|\nabla u_{t}\right\|_{2}}{\|\nabla u\|_{2}} \leq 2(1-\gamma)^{2}\left(\|\nabla u\|_{2}^{2}\right)^{2 \gamma}+\frac{1}{2} \frac{\left\|\nabla u_{t}\right\|_{2}^{2}}{\|\nabla u\|_{2}^{2}} \\
& \leq 2(1-\gamma)^{2}\left(\frac{2(p+1)(\gamma+1)}{p-2 \gamma-1} E(t)\right)^{\frac{2 \gamma}{\gamma+1}}+\frac{1}{2} \frac{\left\|\nabla u_{t}\right\|_{2}^{2}}{\|\nabla u\|_{2}^{2}}
\end{aligned}
$$


and

$$
2 \frac{\int_{\Omega}|u|^{p-1} u u_{t} d x}{\|\nabla u\|_{2}^{2}} \leq 2 B_{1}^{2(p+1)}\left(\frac{2(p+1)(\gamma+1)}{p-2 \gamma-1} E(t)\right)^{\frac{p-1}{\gamma+1}}+\frac{1}{2} \frac{\left\|\nabla u_{t}\right\|_{2}^{2}}{\|\nabla u\|_{2}^{2}} .
$$

Combining these estimates, (3.33) becomes

$$
\begin{aligned}
\frac{d}{d t} H(t)+\left(1-2 B_{1}(\varepsilon H(t))^{\frac{1}{2}}\right) \frac{\left\|\nabla u_{t}\right\|_{2}^{2}}{\|\nabla u\|_{2}^{2}} \\
\leq 2(1-\gamma)^{2}\left(\frac{2(p+1)(\gamma+1)}{p-2 \gamma-1}\right)^{\frac{2 \gamma}{\gamma+1}} E(t)^{\frac{2 \gamma}{\gamma+1}} \\
\quad+2 B_{1}^{2(p+1)}\left(\frac{2(p+1)(\gamma+1)}{p-2 \gamma-1}\right)^{\frac{p-1}{\gamma+1}} E(t)^{\frac{p-1}{\gamma+1}} \\
=c_{8} E(0)^{\frac{2 \gamma}{\gamma+1}}\left(\frac{E(t)}{E(0)}\right)^{\frac{2 \gamma}{\gamma+1}}+c_{9} E(0)^{\frac{p-1}{\gamma+1}}\left(\frac{E(t)}{E(0)}\right)^{\frac{p-1}{\gamma+1}} \\
\leq c_{10}(E(t))^{\frac{2 \gamma}{\gamma+1}},
\end{aligned}
$$

where we have used the fact that $0<E(t) \leq E(0)$ and $p>2 \gamma+1$ on the last inequality with $c_{8}=2(1-\gamma)^{2}\left(\frac{2(p+1)(\gamma+1)}{p-2 \gamma-1}\right)^{\frac{2 \gamma}{\gamma+1}}, c_{9}=2 B_{1}^{2(p+1)}\left(\frac{2(p+1)(\gamma+1)}{p-2 \gamma-1}\right)^{\frac{p-1}{\gamma+1}}$ and $c_{10}=c_{8}+c_{9} E(0)^{\frac{p-2 \gamma-1}{\gamma+1}}$.

If $1-2 B_{1}(\varepsilon H(0))^{\frac{1}{2}}>0$, then there exists $T_{3}$ such that $0<T_{3} \leq T_{1}$ and

$$
1-2 B_{1}(\varepsilon H(t))^{\frac{1}{2}} \geq 0, \quad \text { for } 0 \leq t \leq T_{3} \text {. }
$$

Thus

$$
\frac{d}{d t} H(t) \leq c_{10}(E(t))^{\frac{2 \gamma}{\gamma+1}}
$$

for $0 \leq t \leq T_{3}$. Employing (3.18) with $\omega=\frac{2 \gamma}{\gamma+1}$ gives

$$
H(t) \leq H(0)+\alpha_{2} E(0)^{\frac{\gamma}{\gamma+1}}, \quad \text { for } 0 \leq t \leq T_{3},
$$

with

$$
\alpha_{2}=\frac{(2 \gamma+1) c_{10}}{\gamma+1}\left(\alpha E(0)^{\frac{\gamma}{2(\gamma+1)}}+\beta\right)^{2}
$$

Therefore, we see that (3.34)-(3.36) hold true for $0 \leq t<T_{1}$, which gives the estimate (3.26) for $0<\gamma<1$.

Proposition 3.6 Under the same assumptions as in Proposition 3.5, assume further that the initial data satisfies

$$
\begin{cases}1-2(\gamma+2) B_{1}\left(\varepsilon\left(H(0)+\alpha_{1} E(0)^{\frac{p-2 \gamma}{\gamma+1}}\right)^{\gamma}\right)^{\frac{1}{2}}>0, & \text { if } \gamma \geq 1 \\ 1-2(2 \gamma+1) B_{1}\left(\varepsilon\left(H(0)+\alpha_{2} E(0)^{\frac{\gamma}{\gamma+1}}\right)\right)^{\frac{1}{2}}>0, & \text { if } 0<\gamma<1 .\end{cases}
$$


Then it holds that

$$
\|\nabla u\|_{2}^{2} \geq \alpha_{3}(1+t)^{-\frac{1}{\gamma}}, \quad \text { for } 0 \leq t<T_{1}
$$

where $\alpha_{3}$ is a positive constant.

Proof (i) In the case $\gamma \geq 1$, we obtain from (3.22) with $k=2$ that

$$
\begin{aligned}
& \frac{d}{d t}\left[\varepsilon \frac{\left\|u_{t}\right\|_{2}^{2}}{\|\nabla u\|_{2}^{2(\gamma+2)}}+\frac{1}{\|\nabla u\|_{2}^{2}}\right]+2 \frac{\left\|\nabla u_{t}\right\|_{2}^{2}}{\|\nabla u\|_{2}^{2(\gamma+2)}} \\
& \quad=-2(\gamma+2) \varepsilon \frac{\int_{\Omega} \nabla u \nabla u_{t} d x}{\|\nabla u\|_{2}^{2(\gamma+3)}}\left\|u_{t}\right\|_{2}^{2}-4 \frac{\int_{\Omega} \nabla u \nabla u_{t} d x}{\|\nabla u\|_{2}^{4}}+2 \frac{\int_{\Omega}|u|^{p-1} u u_{t} d x}{\|\nabla u\|_{2}^{2(\gamma+2)}} .
\end{aligned}
$$

As in deriving the estimates for case (i) of Proposition 3.5, we get the following estimates:

$$
\begin{aligned}
& 2(\gamma+2) \varepsilon \frac{\int_{\Omega} \nabla u \nabla u_{t} d x}{\|\nabla u\|_{2}^{2(\gamma+3)}}\left\|u_{t}\right\|_{2}^{2} \leq 2(\gamma+2) B_{1} \varepsilon \frac{\left\|u_{t}\right\|_{2}}{\|\nabla u\|_{2}} \frac{\left\|\nabla u_{t}\right\|_{2}^{2}}{\|\nabla u\|_{2}^{2(\gamma+2)}}, \\
& 4 \frac{\int_{\Omega} \nabla u \nabla u_{t} d x}{\|\nabla u\|_{2}^{4}} \leq \frac{1}{2} \frac{\left\|\nabla u_{t}\right\|_{2}^{2}}{\|\nabla u\|_{2}^{2(\gamma+2)}}+8\|\nabla u\|_{2}^{2(\gamma-1)},
\end{aligned}
$$

and

$$
2 \frac{\int_{\Omega}|u|^{p-1} u u_{t} d x}{\|\nabla u\|_{2}^{2(\gamma+2)}} \leq \frac{1}{2} \frac{\left\|\nabla u_{t}\right\|_{2}^{2}}{\|\nabla u\|_{2}^{2(\gamma+2)}}+2 B_{1}^{2(p+1)}\|\nabla u\|_{2}^{2(p-\gamma-2)} .
$$

Thus, using the above estimates in (3.39), together with the fact that $\varepsilon \frac{\left\|t_{t}\right\|_{2}}{\|\nabla u\|_{2}} \leq\left(\varepsilon H^{\gamma}(t)\right)^{\frac{1}{2}}$ by (3.27) and the estimate (3.25), yield

$$
\begin{aligned}
& \frac{d}{d t}\left[\varepsilon \frac{\left\|u_{t}\right\|_{2}^{2}}{\|\nabla u\|_{2}^{2(\gamma+2)}}+\frac{1}{\|\nabla u\|_{2}^{2}}\right]+\frac{\left\|\nabla u_{t}\right\|_{2}^{2}}{\|\nabla u\|_{2}^{2(\gamma+2)}} \\
& \leq 2(\gamma+2) B_{1}\left(\varepsilon H^{\gamma}(t)\right)^{\frac{1}{2}} \frac{\left\|\nabla u_{t}\right\|_{2}^{2}}{\|\nabla u\|_{2}^{2(\gamma+2)}} \\
& \quad+8\|\nabla u\|_{2}^{2(\gamma-1)}+2 B_{1}^{2(p+1)}\|\nabla u\|_{2}^{2(p-\gamma-2)} \\
& \leq 2(\gamma+2) B_{1}\left(\varepsilon\left(H(0)+\alpha_{1} E(0)^{\frac{p-2 \gamma}{\gamma+1}}\right)^{\gamma}\right)^{\frac{1}{2}} \frac{\left\|\nabla u_{t}\right\|_{2}^{2}}{\|\nabla u\|_{2}^{2(\gamma+2)}} \\
& \quad+8\|\nabla u\|_{2}^{2(\gamma-1)}+2 B_{1}^{2(p+1)}\|\nabla u\|_{2}^{2(p-\gamma-2)},
\end{aligned}
$$

for $0 \leq t<T_{1}$.

If $1-2(\gamma+2) B_{1}\left(\varepsilon\left(H(0)+\alpha_{1} E(0)^{\frac{p-2 \gamma}{\gamma+1}}\right)^{\gamma}\right)^{\frac{1}{2}}>0$, then, using $\|\nabla u\|_{2}^{2} \leq c_{2}(1+t)^{-\frac{1}{\gamma}}$ by (3.17), we see that

$$
\begin{aligned}
\frac{d}{d t}\left[\varepsilon \frac{\left\|u_{t}\right\|_{2}^{2}}{\|\nabla u\|_{2}^{2(\gamma+2)}}+\frac{1}{\|\nabla u\|_{2}^{2}}\right] & \leq c_{11}\left((1+t)^{-\frac{\gamma-1}{\gamma}}+(1+t)^{-\frac{p-\gamma-2}{\gamma}}\right) \\
& \leq 2 c_{11}(1+t)^{-\lambda_{1}}=2 c_{11}(1+t)^{-\frac{\gamma-1}{\gamma}}
\end{aligned}
$$


and

$$
\varepsilon \frac{\left\|u_{t}\right\|_{2}^{2}}{\|\nabla u\|_{2}^{2(\gamma+2)}}+\frac{1}{\|\nabla u\|_{2}^{2}} \leq c_{12}(1+t)^{\frac{1}{\gamma}}
$$

which implies that

$$
\|\nabla u\|_{2}^{2} \geq c_{13}(1+t)^{-\frac{1}{\gamma}}, \quad \text { for } 0 \leq t<T_{1},
$$

with $\lambda_{1}=\min \left\{\frac{\gamma-1}{\gamma}, \frac{p-\gamma-2}{\gamma}\right\}=\frac{\gamma-1}{\gamma}$ and some positive constants $c_{i}, i=11-13$.

(ii) In the case $0<\gamma<1$, it follows from (3.22) with $k=1+\gamma$ that

$$
\begin{aligned}
& \frac{d}{d t}\left[\varepsilon \frac{\left\|u_{t}\right\|_{2}^{2}}{\|\nabla u\|_{2}^{2(2 \gamma+1)}}+\frac{1}{\|\nabla u\|_{2}^{2 \gamma}}\right]+2 \frac{\left\|\nabla u_{t}\right\|_{2}^{2}}{\|\nabla u\|_{2}^{2(2 \gamma+1)}} \\
& =-2(2 \gamma+1) \varepsilon \frac{\int_{\Omega} \nabla u \nabla u_{t} d x}{\|\nabla u\|_{2}^{2(2 \gamma+2)}}\left\|u_{t}\right\|_{2}^{2}-2(\gamma+1) \frac{\int_{\Omega} \nabla u \nabla u_{t} d x}{\|\nabla u\|_{2}^{2(\gamma+1)}} \\
& \quad+2 \frac{\int_{\Omega}|u|^{p-1} u u_{t} d x}{\|\nabla u\|_{2}^{2(2 \gamma+1)}} .
\end{aligned}
$$

Estimate the right-hand side of (3.40) as in the case (ii) of Proposition 3.5 to obtain

$$
\begin{aligned}
& 2(2 \gamma+1) \varepsilon \frac{\int_{\Omega} \nabla u \nabla u_{t} d x}{\|\nabla u\|_{2}^{2(2 \gamma+2)}}\left\|u_{t}\right\|_{2}^{2} \leq 2(2 \gamma+1) B_{1}(\varepsilon H(t))^{\frac{1}{2}} \frac{\left\|\nabla u_{t}\right\|_{2}^{2}}{\|\nabla u\|_{2}^{2(2 \gamma+1)}}, \\
& 2(\gamma+1) \frac{\int_{\Omega} \nabla u \nabla u_{t} d x}{\|\nabla u\|_{2}^{2(\gamma+1)}} \leq 2(\gamma+1)^{2}+\frac{1}{2} \frac{\left\|\nabla u_{t}\right\|_{2}^{2}}{\|\nabla u\|_{2}^{2(2 \gamma+1)}},
\end{aligned}
$$

and

$$
\frac{2 \int_{\Omega}|u|^{p-1} u u_{t} d x}{\|\nabla u\|_{2}^{2(2 \gamma+1)}} \leq 2 B_{1}^{2(p+1)}\|\nabla u\|_{2}^{2(p-2 \gamma-1)}+\frac{1}{2} \frac{\left\|\nabla u_{t}\right\|_{2}^{2}}{\|\nabla u\|_{2}^{2(2 \gamma+1)}} .
$$

Back to (3.40), employing these estimates and using (3.26), we deduce that

$$
\begin{aligned}
& \frac{d}{d t}\left[\varepsilon \frac{\left\|u_{t}\right\|_{2}^{2}}{\|\nabla u\|_{2}^{2(2 \gamma+1)}}+\frac{1}{\|\nabla u\|_{2}^{2 \gamma}}\right]+\frac{\left\|\nabla u_{t}\right\|_{2}^{2}}{\|\nabla u\|_{2}^{2(2 \gamma+1)}} \\
& \leq 2(2 \gamma+1) B_{1}(\varepsilon H(t))^{\frac{1}{2}} \frac{\left\|\nabla u_{t}\right\|_{2}^{2}}{\|\nabla u\|_{2}^{2(2 \gamma+1)}} \\
& \quad+2(\gamma+1)^{2}+2 B_{1}^{2(p+1)}\left(\|\nabla u\|_{2}^{2}\right)^{(p-2 \gamma-1)} \\
& \leq 2(2 \gamma+1) B_{1}\left(\varepsilon\left(H(0)+\alpha_{2} E(0)^{\frac{\gamma}{\gamma+1}}\right)\right)^{\frac{1}{2}} \frac{\left\|\nabla u_{t}\right\|_{2}^{2}}{\|\nabla u\|_{2}^{2(2 \gamma+1)}} \\
& \quad+2(\gamma+1)^{2}+2 B_{1}^{2(p+1)}\left(\|\nabla u\|_{2}^{2}\right)^{(p-2 \gamma-1)}
\end{aligned}
$$

for $0 \leq t<T_{1}$.

If $1-2(2 \gamma+1) B_{1}\left(\varepsilon\left(H(0)+\alpha_{2} E(0)^{\frac{\gamma}{\gamma+1}}\right)\right)^{\frac{1}{2}}>0$, then, by $\|\nabla u\|_{2}^{2} \leq c_{2}(1+t)^{-\frac{1}{\gamma}}$, we have

$$
\frac{d}{d t}\left[\varepsilon \frac{\left\|u_{t}\right\|_{2}^{2}}{\|\nabla u\|_{2}^{2(2 \gamma+1)}}+\frac{1}{\|\nabla u\|_{2}^{2 \gamma}}\right] \leq 2(\gamma+1)^{2}+c_{14}(1+t)^{-\frac{p-2 \gamma-1}{\gamma}} \leq c_{15},
$$


and

$$
\varepsilon \frac{\left\|u_{t}\right\|_{2}^{2}}{\|\nabla u\|_{2}^{2(2 \gamma+1)}}+\frac{1}{\|\nabla u\|_{2}^{2 \gamma}} \leq c_{16}(1+t)
$$

for $0 \leq t<T_{1}$ with some positive constants $c_{i}, i=14-16$. This implies the desired estimate (3.38) for $0<\gamma<1$.

Now, we are ready to state and prove our main result.

Theorem 3.7 Let $\left(u_{0}, u_{1}\right) \in H^{2}(\Omega) \cap H_{0}^{1}(\Omega) \times L^{2}(\Omega), I(0)>0$ and (3.5)-(3.6) hold. Then the solution $u$ of problem (1.1)-(1.3) satisfies

$$
\|\nabla u\|_{2}^{2} \leq \alpha_{4}(1+t)^{-\frac{1}{\gamma}} \quad \text { and } \quad\left\|u_{t}\right\|_{2}^{2} \leq \alpha_{5}(1+t)^{-2-\frac{1}{\gamma}} \quad \text { for } t \geq 0 .
$$

Moreover, suppose that the initial data satisfies $u_{0} \neq 0$ and

$$
\begin{cases}1-2(\gamma+2) B_{1}\left(\varepsilon\left(H(0)+\alpha_{1} E(0)^{\frac{p-2 \gamma}{\gamma+1}}\right)^{\gamma}\right)^{\frac{1}{2}}>0, & \text { if } \gamma \geq 1 ; \\ 1-6 B_{1}\left(\varepsilon\left(H(0)+\alpha_{2} E(0)^{\frac{\gamma}{\gamma+1}}\right)\right)^{\frac{1}{2}}>0, & \text { if } 0<\gamma<1 .\end{cases}
$$

Then

$$
\|\nabla u\|_{2}^{2} \geq \alpha_{6}(1+t)^{-\frac{1}{\gamma}}, \quad \text { for } t \geq 0
$$

with some positive constants $\alpha_{i}, i=4,5,6$.

Proof Thanks to the decay estimates (3.11) and (3.17), we obtain (3.41). Setting

$$
T_{1}=\sup \left\{t \in[0, \infty) \mid\|\nabla u(s)\|_{2}^{2}>0 \text { for } 0 \leq s<t\right\}
$$

then, we see that $T_{1}>0$ and $\|\nabla u(t)\|_{2}^{2}>0$ for $0 \leq t<T_{1}$, because of $\left\|\nabla u_{0}\right\|_{2}>0$. If $T_{1}<\infty$, then it holds that

$$
\left\|\nabla u\left(T_{1}\right)\right\|_{2}^{2}=0
$$

However, from $\|\nabla u\|_{2}^{2} \geq \alpha_{3}(1+t)^{-\frac{1}{\gamma}}$ by (3.38), we observe that

$$
\lim _{t \rightarrow T_{1}}\|\nabla u(t)\|_{2}^{2} \geq C\left(1+T_{1}\right)^{-\frac{1}{\gamma}}>0
$$

which contradicts (3.44). Hence, we have $T_{1}=\infty$ and $\|\nabla u(t)\|_{2}^{2}>0$ for all $t \geq 0$. Therefore, from (3.38), we have

$$
\|\nabla u(t)\|_{2}^{2} \geq \alpha_{6}(1+t)^{-\frac{1}{\gamma}}, \quad \text { for } t \geq 0
$$

which gives the decay estimate (3.44). 


\section{Competing interests}

The author declares that they have no competing interests.

\section{Acknowledgements}

This work was funded by National Taipei University of Technology under the project 101T458-13.

Received: 14 March 2012 Accepted: 9 August 2012 Published: 30 August 2012

\section{References}

1. Kirchhoff, G: Vorlesungen über Mechanik. Teubner, Leipzig (1883)

2. Hosoys, M, Yamada, Y: On some nonlinear wave equations II: global existence and energy decay of solutions. J. Fac. Sci., Univ. Tokyo, Sect. 1A, Math. 38, 239-250 (1991)

3. Nishihara, K, Yamada, Y: On global solutions of some degenerate quasilinear hyperbolic equations with dissipative terms. Funkc. Ekvacioj 33, 151-159 (1990)

4. Nakao, M: A difference inequality and its application to nonlinear evolution equations. J. Math. Soc. Jpn. 30, 747-762 (1978)

5. Nishihara, K: Decay properties of solutions of some quasilinear hyperbolic equations with strong damping. Nonlinear Anal. 21, 17-21 (1993)

6. Nishihara, K, Ono, K: On a nonlinear degenerate integro-differential equation of hyperbolic type with a strong dissipation. Adv. Math. Sci. Appl. 5(2), 457-476 (1995)

7. Ono, K: Global existence and decay properties of solutions for some mildly degenerate nonlinear dissipative Kirchhoff strings. Funkc. Ekvacioj 40(2), 255-270 (1997)

8. Ono, K: On global existence, asymptotic stability and blowing up of solutions for some degenerate nonlinear wave equations of Kirchhoff type with strong dissipation. Math. Methods Appl. Sci. 20, 151-177 (1997)

9. Ono, K: On decay properties of solutions for degenerate strongly damped wave equations of Kirchhoff type. J. Math. Anal. Appl. 381(1), 229-239 (2011)

doi:10.1186/1687-2770-2012-93

Cite this article as: Wu: On decay properties of solutions for degenerate Kirchhoff equations with strong damping and source terms. Boundary Value Problems 2012 2012:93.

\section{Submit your manuscript to a SpringerOpen ${ }^{\odot}$ journal and benefit from:}

- Convenient online submission

- Rigorous peer review

Immediate publication on acceptance

- Open access: articles freely available online

- High visibility within the field

- Retaining the copyright to your article 